\title{
In vitro hydrodynamics, cusp-bending deformation, and root distensibility for different types of aortic valve-sparing operations: Remodeling, sinus prosthesis, and reimplantation
}

\author{
Armin Erasmi, MD, Hans-H. Sievers, MD, Michael Scharfschwerdt, Thorsten Eckel, and Martin Misfeld, MD, PhD
}

Objective: Preserving aortic valve cusps during operations for aortic root pathology theoretically offers several advantages over alternative prosthetic valve-bearing conduits. Functional properties of different valve-sparing techniques under defined conditions are not well studied.

Methods: Fresh porcine aortic roots were investigated in a pulsatile flow simulator, either native root or after different types of valve-sparing procedures (remodeling, sinus prosthesis, and reimplantation). Functional parameters, such as transvalvular pressure gradient, closing volume, cusp-bending deformation, and distensibility at different levels of the root were analyzed.

Results: The mean pressure gradient was highest in reimplantation techniques (8.4 $\pm 1.8 \mathrm{~mm} \mathrm{Hg})$ compared with sinus prostheses $(7.2 \pm 0.9 \mathrm{~mm} \mathrm{Hg}, P=.01)$ and remodeling techniques $(6.8 \pm 1.0 \mathrm{~mm} \mathrm{Hg}, P=.002)$, mirror imaging the closing volume (reimplantation, $1.5 \pm 0.4 \mathrm{~mL}$; sinus prostheses, $2.3 \pm 0.7 \mathrm{~mL}[P<.001]$; remodeling, $3.4 \pm 1.1 \mathrm{~mL}[P<.001])$. Bending deformation indices increased significantly from remodeling $(0.45 \pm 0.05)$ and sinus prostheses $(0.58 \pm 0.06)$ to reimplantation techniques $(0.73 \pm 0.09)$. Dynamic changes in area of all techniques were decreased at the sinotubular junction and the commissural and sinus levels when compared with those seen in native roots but increased at the annular level for techniques with unfixed annulus (remodeling and modified sinus prosthesis).

From the Department of Cardiac Surgery, University Clinic of Schleswig-Holstein, Campus Luebeck, Luebeck, Germany.

Received for publication April 19, 2005; revisions received June 3, 2005; accepted for publication June 7, 2005.

Address for reprints: Hans-H. Sievers, MD, Department of Cardiac Surgery, University Clinic of Schleswig-Holstein, Campus Luebeck, Ratzeburger Allee 160, 23538 Luebeck, Germany (E-mail: herzchir@ medinf.mu-luebeck.de).

J Thorac Cardiovasc Surg 2005; 130:1044-9

$0022-5223 / \$ 30.00$

Copyright $\odot 2005$ by The American Association for Thoracic Surgery

doi:10.1016/j.jtcvs.2005.06.005

Conclusions: In vitro the various aortic valve-sparing operations differed characteristically in their ability to spare valve function, none of them completely meeting native valve behavior. The remodeling techniques exhibited valve dynamics closest to those of the native aortic root. The more the aortic valve is fixed with noncompliant prosthetic material, the more the native root dynamics are impaired.

$\mathrm{T}$ The aortic valve is composed of 3 cusps inserting into the aortic wall in 3 crown-shaped fibrous rings that confine the sinuses of Valsalva downstream and the intervalvular trigones upstream. The commissures, as the top of the adjoining fibrous rings, border on the sinotubular junction, whereas the so-called aortic annulus, which represents the transition area between the left ventricle and the aorta, includes the nadirs of the sinuses, the muscular and the membranous septum, and the aortomitral curtain. All of these functional structures perform very sophisticated cyclic movements, interacting with each other. ${ }^{1}$ Their dynamic characteristics are aimed at reducing stress on the cusps and optimizing ventricular-arterial coupling to warrant hemodynamic efficiency without structural deterioration of the cusps throughout life. Aneurysms and dissections involving the aortic root lead to morphologic, geometric, and hemodynamic abnormalities that often leave the cusps macroscopically intact. Valve-sparing surgical techniques have been developed, 


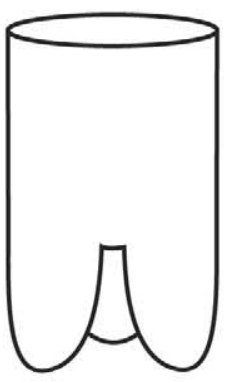

a

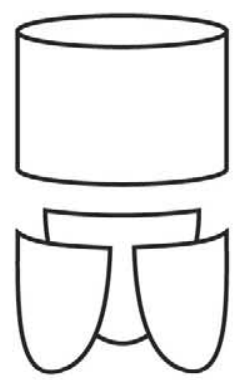

b

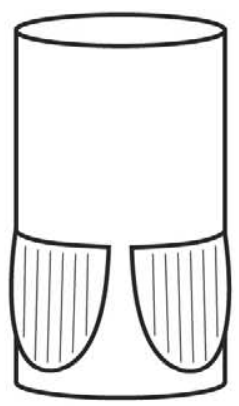

C

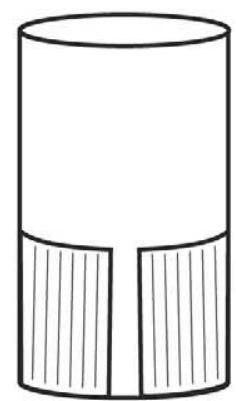

d

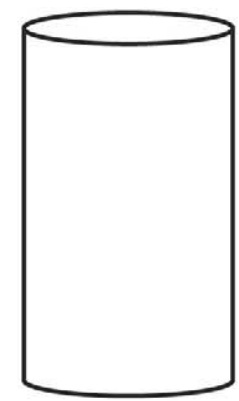

e

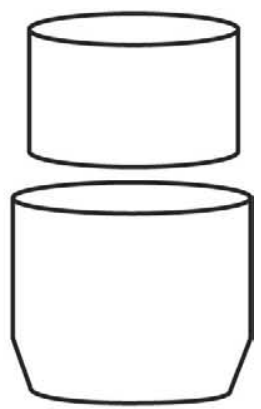

f

Figure 1. Schematic drawings of the different valve-sparing techniques. Left to right: remodeling, modified remodeling, sinus prosthesis, modified sinus prosthesis, reimplantation, and modified reimplantation.

driven by the desire to preserve the benefits of native structures and dynamics, as well as to avoid the complications of alternative and commonly used prosthetic valve substitutes. Different valve-sparing techniques are applied, varying from prosthetic replacement of the sinuses of Valsalva only with the remodeling technique ${ }^{2}$ to total replacement of the wall of the aortic root with a tubular Dacron graft with the reimplantation technique. ${ }^{3}$ Although clinical results of both techniques have been reported to be excellent, ${ }^{4,5}$ there is a lack of comparison of functional characteristics of the different valve-sparing techniques under defined and reproducible conditions.

\section{Materials and Methods}

Two different aortic valve-sparing principles were investigated: the remodeling and the reimplantation techniques. With the remodeling technique, 2 different tube designs (remodeling and modified remodeling) were tested, and 4 tube designs (sinus and modified sinus prosthesis, reimplantation, and modified reimplantation) were tested with the reimplantation technique (Figure 1) and compared with the native aortic roots of pigs.

Aortic roots were carefully dissected from pig hearts from a local slaughterhouse within 4 hours of slaughter. The ascending aorta was cut away up to $1 \mathrm{~cm}$ above the sinotubular junction. The left ventricular muscle and the anterior mitral valve leaflet were removed, and a Dacron tube $(26 \mathrm{~mm}$ in diameter and $1.5 \mathrm{~cm}$ in length) was sutured to these structures for mounting the root in the test circuit, leaving roughly $1 \mathrm{~cm}$ of tissue between this prosthetic mounting tube and the aortic annulus.

\section{Surgical Techniques}

For each technique, the size of the Dacron tube (Boston Scientific Corp, Wayne, NJ) was calculated by multiplying the measured outer diameter of the unloaded sinotubular junction by 1.2 to roughly achieve the diameter at systemic pressure. ${ }^{6}$ The sizes of the prostheses as thus calculated were 30 to $32 \mathrm{~mm}$ in all procedures.

Figure 1 illustrates the principles of the different surgical procedures. In all aortic roots, the 3 sinuses of Valsalva were resected, leaving a rim of sinus tissue of $2 \mathrm{~mm}$ to the fibrous ring of the cusps. For the remodeling technique, the Dacron tube was trimmed in a 3-tongue fashion for replacement of the sinuses (Figure $1, a$ ). The graft was sewn on the remaining aortic root by using a continuous 4-0 Prolene suture (Ethicon, Norderstedt, Germany). The remodeling technique was modified by sewing the 3 tongues separately into the resected sinuses, enabling more precise matching and easier surgical handling of the sinus reconstruction (Figure 1, b). Finally, the remaining Dacron tube was sewn onto the reconstructed root at the height of the sinotubular junction. The sinus prosthesis was self-manufactured. A Dacron tube was trimmed by inserting sinus of Valsalva-shaped pieces of the same tube into the graft for creation of the sinuses. The 3 neosinuses were inserted by turning the prosthetic material at a $90^{\circ}$ angle to use the distensibility of the Dacron material in circumferential direction (Figure 1,c). The aortic valve was implanted into this prosthesis by using interrupted, U-shaped 4-0 Prolene sutures at the annulus in a planar fashion and a continuous 4-0 Prolene suture for attachment of the valve from inside the prosthesis. The sinus prosthesis was further modified by inserting 3 square pieces of the same graft into the tube after turning them by $90^{\circ}$, with no rim of the Dacron tube left at the base of the sinus (Figure 1,d). This prosthesis enables the surgeon to adapt the height of the graft to the varying heights of the commissures of the pig aortic root. The aortic valve was then inserted into the prosthesis, as described for the sinus prosthesis.

For the original reimplantation technique, the aortic valve was implanted into an untrimmed Dacron tube (Figure 1,e), as described above. For the modified reimplantation technique (Figure $1, f$ ), a 6-mm-larger graft was downsized to the calculated diameter during the proximal anastomosis by using mattress Teflon feltpledgeted sutures placed in a horizontal plane at the annulus. The valve was then implanted from inside the Dacron tube with 4-0 Prolene continuous sutures. At the sinotubular junction, another graft tailored to the calculated diameter was sutured to the larger proximal prosthesis.

\section{Test Circuit}

The aortic roots were tested in a pulsatile flow simulator, details of which have been described previously. ${ }^{7}$ In brief, the flow simulator 


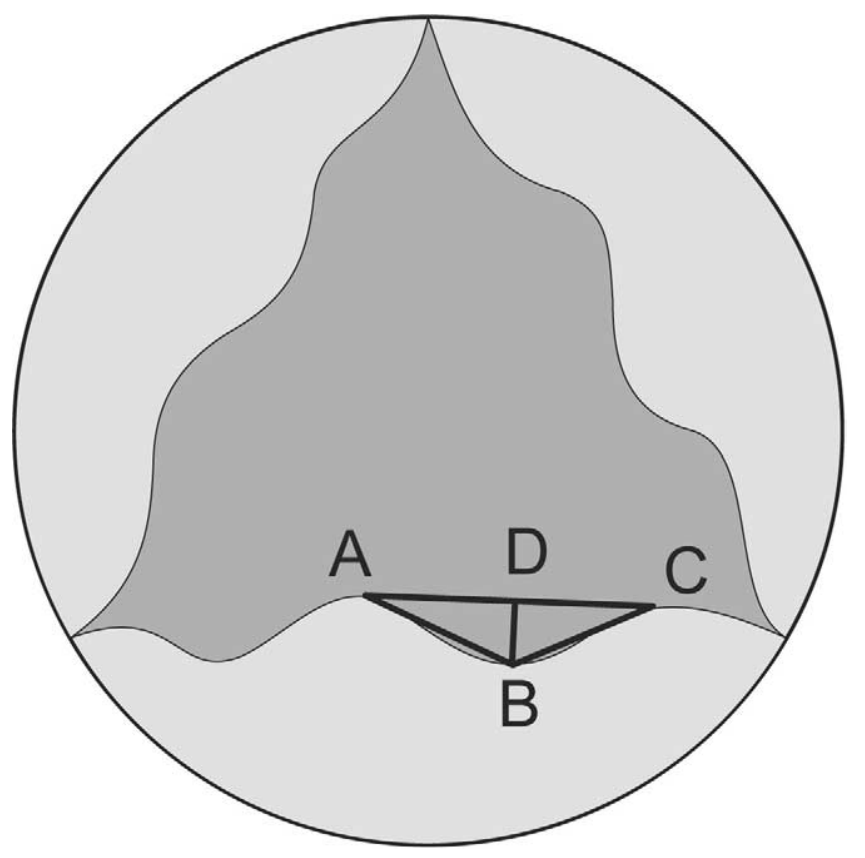

Figure 2. Calculation of the open cusp-bending deformation index. Bending deformation index is defined as fold depth divided by the distance $B C$.

consists of an open atrial reservoir, a cam-plate-driven piston pump with low mass inflow valves, and a compliance simulating ventricular impedance. The aortic root section has a ventricleshaped inflow configuration and an aortic impedance including a height-variable fluid column, an adjustable aortic air compliance chamber, and a special elastoresistive element for peripheral resistance.

The roots were mounted in a fluid reservoir, fixed between 2 spigots in the vertical position, and then tested at a heart rate of 64 cycles per minute, with a stroke volume of $54 \mathrm{~mL}$ for a systemic pressure of $125 / 80 \mathrm{~mm} \mathrm{Hg}$. For each valve-sparing technique, the dynamic function and cusp configuration of at least 6 aortic roots were investigated and compared with those of native aortic roots. Data were collected from 5 consecutive cycles each, and measurements were repeated 10 times for each root and test condition.

\section{Data Acquisition and Analysis}

Pressure measurements were performed by using Envec Ceracore M pressure transducers (Endress + Hauser, Maulburg, Germany) at the inflow and outflow spigots. The flow through the valves was measured with a HT207 ultrasonic flow-meter (Transonic Systems Inc, Ithaca, NY) positioned upstream of the valve. Data were collected digitally at a rate of 500 samples per second. Mean pressure gradient and closing volume from 5 cycles were determined as required in the ISO $5840^{\circ}$ and calculated from the data by using a commercial spreadsheet analysis program.

Movements of the cusps were recorded with a Motionscope HR-1000 high-speed camera (Redlake Imaging Corp, Morgan Hill, Calif) positioned straight above the valve at a rate of 500

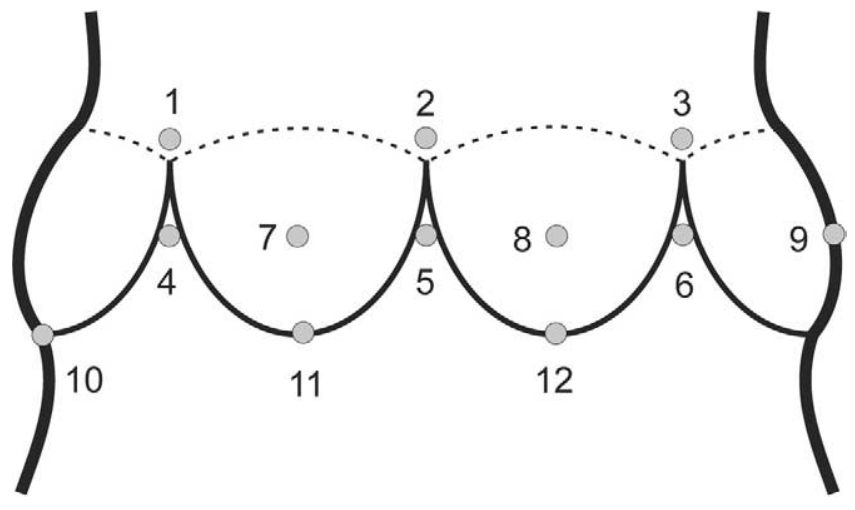

Figure 3. Schematic drawing of the aortic root showing the placement of the ultrasonic microprobes. Probes were placed at the sinotubular junction (1-3), between the crown-shaped fibrous rings for cusp attachment at the beginning of the commissural area (4-6), in the middle of each sinus (7-9), and at the annular level (10-12).

frames per second. Video data were digitized and analyzed with a custom-made motion evaluation software. Open cusp-bending deformations were determined from the still image in midsystole, where the strongest deformation was observed, and quantified by taking 3 points along the edges of the largest fold (Figure 2). The ratio of the maximal fold depth and the shorter distance $\mathrm{BC}$ was used as a cusp-bending deformation index. ${ }^{9}$

Ultrasonic micrometric transceiver-receiver crystals (Sonometrics Corp, London, Ontario, Canada) were used to measure distances at the sinotubular junction, commissural, midsinus, and annular levels, respectively. Figure 3 depicts the position of the probes at the aortic roots. In areas in which the aortic roots were replaced with Dacron material, the probes had to be inserted inside the graft. Cross-sectional areas at each level were calculated from the triangle defined by the distances between the corresponding crystals at peak systolic and end-diastolic pressures, representing roughly maximal and minimal areas. Root distensibility was presented as the total percentage change of area referenced to the value at end diastole.

\section{Statistical Analysis}

Statistical analysis was performed by means of multiple comparisons of the means with 1-way analysis of variance, and the Bonferroni adjustment for multiple tests was used for significant differences. Data were expressed as means \pm the standard deviation of the mean.

\section{Results}

Transvalvular Pressure Gradient and Closing Volume Mean transvalvular pressure gradients and closing volumes are depicted in Figure 4. The reimplantation technique caused significantly higher pressure gradients compared with all other techniques and the native aortic root $(8.85 \pm$ $2.03 \mathrm{~mm} \mathrm{Hg}, P<.001)$. Mean pressure gradients of the modified reimplantation technique $(7.66 \pm 0.9 \mathrm{~mm} \mathrm{Hg})$, 


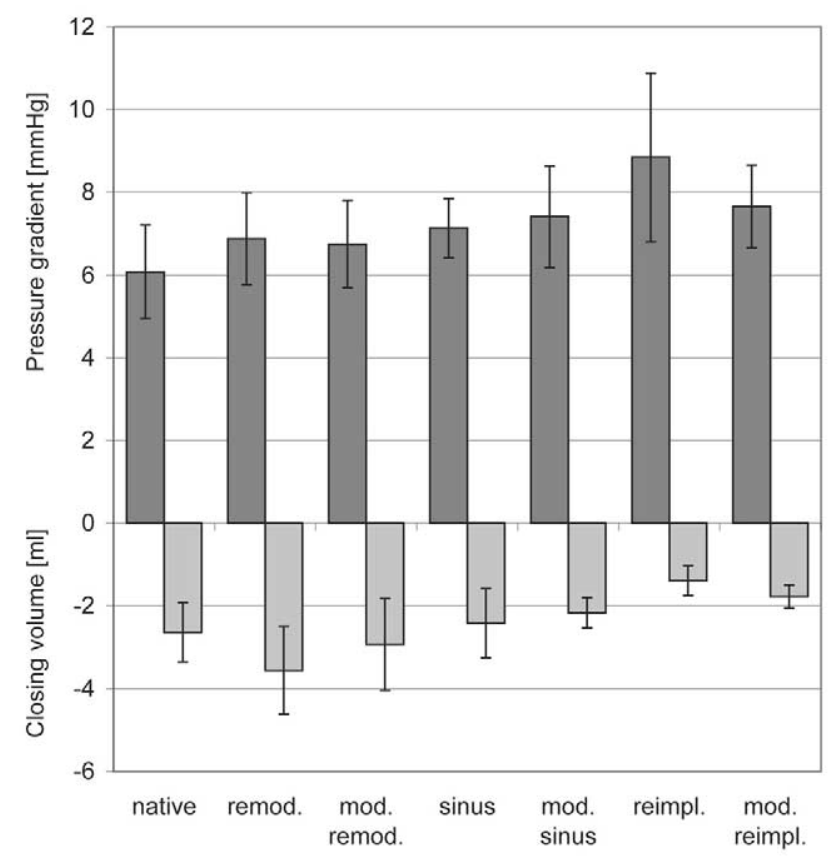

Figure 4. Mean pressure gradients (upper columns) and closing volumes (lower columns) of the different valve-sparing techniques.

sinus prosthesis $(7.14 \pm 0.71 \mathrm{~mm} \mathrm{Hg}, P=.003)$, and modified sinus prosthesis $(7.41 \pm 1.22 \mathrm{~mm} \mathrm{Hg}, P=.019)$ were also significantly higher compared with that of the native aortic root $(6.08 \pm 1.13 \mathrm{~mm} \mathrm{Hg})$.

The different surgical techniques showed only minor differences with regard to the closing volume. Only the reimplantation and the modified reimplantation techniques produced closing volumes significantly lower than those of the other techniques and the native aortic root (reimplantation, $1.39 \pm 0.36 \mathrm{~mL}$; modified reimplantation, $1.77 \pm 0.28$ $\mathrm{mL}$; native, $2.64 \pm 0.72 \mathrm{~mL} ; P<.001)$. Leakage was not observed in any technique.

\section{Bending Deformation Index}

Concerning bending deformation, none of the surgical procedures was comparable with the native aortic root, and all techniques differed significantly from each other (Figure 5). Bending deformation indices for all valve-sparing techniques were more than twice those of the native aortic root and increased in relation to the degree the root was fixed with synthetic noncompliant material.

\section{Changes of Area at Different Levels of the Aortic Root} Aortic root distensibility, expressed as diastolic-to-systolic change of area, decreased in all surgical procedures compared with that of the native aortic root (Figure 6). The cyclic changes of cross-sectional areas were significantly

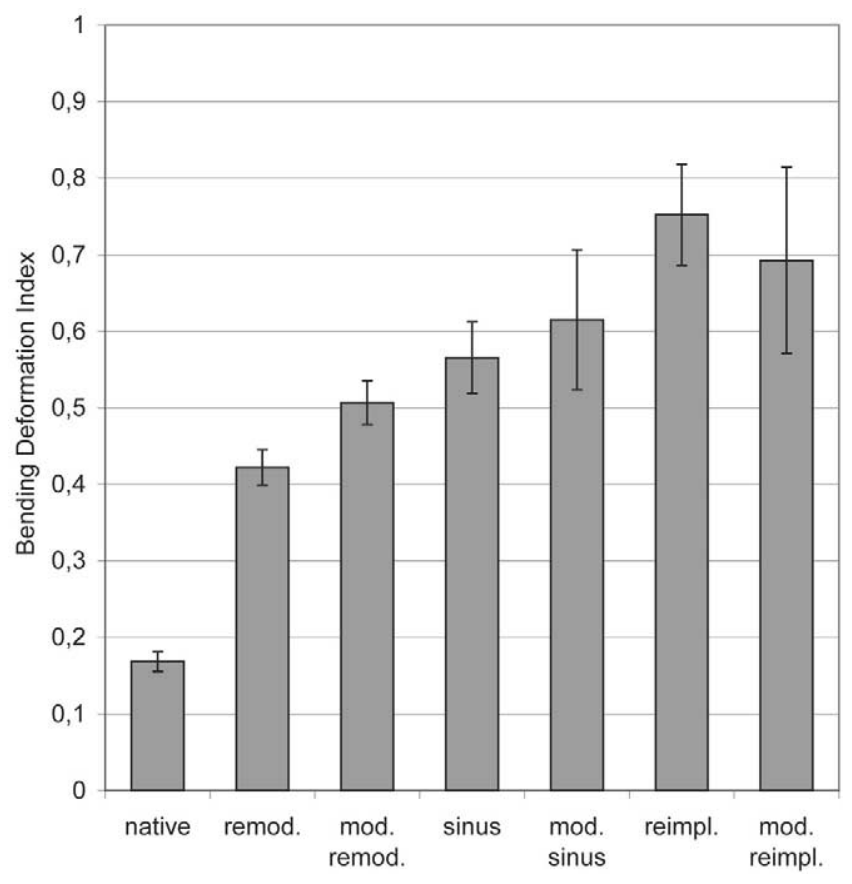

Figure 5. Maximal bending deformation index of the cusps for different valve-sparing techniques.

more pronounced for the remodeling and modified remodeling techniques and also for the sinus prosthesis at the sinotubular junction compared with the reimplantation and modified reimplantation techniques and the modified sinus prosthesis. At the commissural level, the reimplantation and the modified reimplantation techniques had the lowest values $(6.0 \% \pm 1.6 \%$ and $3.94 \% \pm 1.2 \%$, respectively). Distensibility was greater for the sinus and modified sinus prosthesis $(9.2 \% \pm 2.6 \%$ and $10.4 \% \pm 4.3 \%, P<.02)$ and even more for the remodeling and modified remodeling techniques $(17.2 \% \pm 5.1 \%$ and $17.2 \% \pm 6.4 \%, P<.01)$. At the sinus level, no differences were observed between the surgical procedures, and for area changes of less than $5 \%$ during the cardiac cycle, none of the techniques were distensible.

Differences in distensibility between the surgical techniques could be found at the annular level. Although the reimplantation techniques and sinus prosthesis were comparable with the native root, a significant increase of annular distensibility was noted for the remodeling $(9.88 \% \pm 5.4 \%$, $P=.026)$ and modified remodeling $(8.71 \% \pm 4.3 \%, P=$ $.017)$ techniques, as well as for the modified sinus prosthesis $(8.68 \% \pm 2.2 \%, P=.009)$, compared with the native root $(5.16 \% \pm 1.82 \%)$.

\section{Discussion}

This study serves to characterize aortic valve function in a mock circulation after different valve-sparing techniques. 


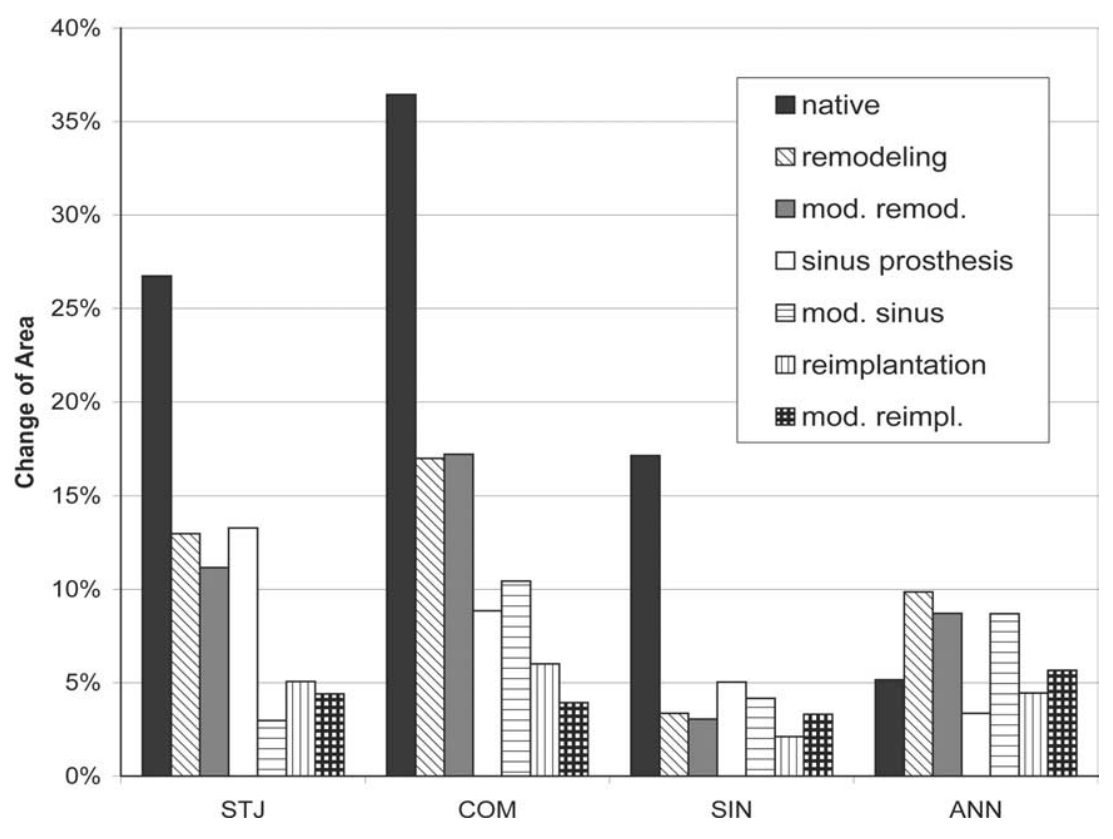

Figure 6. Root distensibility of different valve-sparing techniques. Columns represent the cross-sectional cyclic change of area in the aortic root at the 4 different levels (see Figure 3).

The surgical techniques investigated include the commonly used remodeling technique described by Fagan and colleagues, ${ }^{2}$ the reimplantation technique described by David and Feindel, ${ }^{3}$ modifications of these techniques, and sinus prostheses. The various techniques showed characteristic functional parameters with a tendency toward gradual impairment of the physiologic function caused by increased artificial restriction of root distensibility.

The transvalvular pressure gradient was highest with the reimplantation techniques. Because the diameter of the implanted prosthesis was the same for all techniques investigated, other parameters, like the increased cusp-bending deformation and decreased root distensibility at the sinotubular junction and commissural level, could have contributed to these results. It is also possible that reimplanting the valve into the prosthesis slightly narrowed the annulus, leading to somewhat higher pressure gradients. Some increased pressure gradients could also be found in the clinical situation measured at rest in patients who had undergone the reimplantation technique. However, these findings do not seem to have clinical relevance in the midterm followup. ${ }^{4}$ Nevertheless, long-term follow-up and exercise studies are of interest to further clarify this item.

The closing volume roughly mirrored the transvalvular pressure gradient, which differed significantly from that of the native root only for the reimplantation and modified reimplantation techniques and should have no clinical effect because the absolute volume is small compared with the stroke volume. Whether a causative relation exists between pressure gradient and closing volume remains to be established.
The bending deformation index was significantly increased with the reimplantation techniques and sinus prostheses compared with that seen in the remodeling techniques and native root, which is probably related to the restricted distensibility at the sinotubular and commissural level of these implantation techniques more than the configuration of the sinus. This is supported by the finding that bending deformation was also increased in the modified sinus prosthesis and the modified reimplantation technique, which provide some kind of neosinus. However, there are some other modifications of the reimplantation technique with more prominent neosinuses not investigated in this study. Whether these nonphysiologic bending stresses on the cusps during systole accelerate the cusp-degeneration process ${ }^{10}$ and thus affect the durability of the valve remains to be investigated and seem to have at least no clinical significance in the mid-term follow up. In this context Sripathi and associates ${ }^{11}$ found an unsymmetric opening of wrinkled aortic cusps in a stiff root with increased compressive stresses that might promote the degeneration process. Furukawa and coworkers $^{12}$ also found experimentally and Leyh and colleagues ${ }^{13}$ found clinically that the cusp motion characteristics might be better preserved with the remodeling compared with the reimplantation technique, supporting the hypothesis of better-preserved valve function with the remodeling technique. Further investigations, also including opening and closing velocities, will be needed.

Regarding distensibility at the same area in the root, namely at the sinus and commissural levels, we found a reduced change of area that was pronounced in the reimplantation and modified reimplantation techniques both for 
the sinus and commissural levels but somewhat preserved for the commissural level with the remodeling techniques and sinus prostheses. Whether this was related to the more distensible native intervalvular tissue in the remodeling techniques, the stretchable prosthetic material in the sinus prostheses, or the cyclic change of cross-sectional configuration from the sinus shape to the circular shape remains unknown. Interestingly, the distensibility at the annular level was increased compared with that seen in the native root with the remodeling techniques and with the modified sinus prosthesis. Because the preparation techniques at the annular level were identical for all procedures, this finding remains difficult to understand. Whether this finding is related to a somewhat nonphysiologic increase in wall tension at the annular level as a response to restricted distensibility downstream remains to be determined. But only these techniques preserve the potential for unrestricted dilatation of the annulus, which the fixed annulus by prosthetic tube in the other techniques does not. Nevertheless, in 2 reoperations after the remodeling technique, we found the newly developed aortic insufficiency to be caused by an outward movement of the base of the sinuses, rendering the cusps noncoapting. Further clinical and experimental studies on this matter are necessary.

It needs to be stated that on the basis of our results, the techniques that imitate the hemodynamics of the native aortic root most adequately are the remodeling and the modified remodeling techniques. In certain pathologies, however, as in acute type A aortic dissection with enlarged roots and more definitely in Marfan syndrome, surgical techniques that stabilize the aortic root at the annular level and prevent it from further dilatation should be considered. However, only prospective randomized studies will show which procedure offers the best long-term results for which group of patients.

There are some limitations to this study. First, in vivo results could well be different from those of this in vitro study with nonliving subannular material. This potential drawback, however, could also be an advantage because it could unmask effects that are not quite so clear when living subannular tissue, like septal muscle, partially compensates for increased procedural wall tension at the annular level. Second, the retained tissue of the porcine root has functional characteristics that differ from those of the diseased tissue of a patient; however, because most of the tissue is replaced with prosthetic material, this possible effect is minimized. Third, we did not use blood but rather saline water, which has different viscosity features that might have influenced valve-bending deformation. Finally, alterations in distensibility caused by, for example, pseudoneointima proliferation could not be evaluated in this study.

\section{References}

1. Yacoub MH, Kilner PJ, Birks EJ, Misfeld M. The aortic outflow and root: a tale of dynamism and crosstalk. Ann Thorac Surg. 1999;68: S37-43.

2. Fagan A, Yacoub MH, Pillai R, Radley-Smith R. Dacron replacement of the ascending aorta and sinuses with resuspension of the aortic valve and re-implantation of the coronary arteries: a new method for treatment of aneurysmal or acute dissection of the aortic root. Proceedings of the Joint International Cardiovascular and Thoracic Surgical Conference, Stockholm [abstract]. Scand J Cardiothorac Surg. 1982;16: 175

3. David TE, Feindel CM. An aortic valve sparing operation for patients with aortic incompetence and aneurysm of the ascending aorta. J Thorac Cardiovasc Surg. 1992;103:617-22.

4. Leyh RG, Schmidtke C, Bartels C, Sievers HH. Valve-sparing aortic root replacement (remodeling/reimplantation) in acute type A dissection. Ann Thorac Surg. 2000;70:21-4.

5. Bethea BT, Fitton TP, Alejo DE, Barreiro CJ, Cattaneo SM, Dietz HC, et al. Results of aortic valve-sparing operations: experience with remodeling and reimplantation procedures in 65 patients. Ann Thorac Surg. 2004;78:767-72.

6. Hansen B, Menkis AH, Vesely I. Longitudinal and radial distensibility of porcine aortic root. Ann Thorac Surg. 1995;60:S384-90.

7. Scharfschwerdt M, Misfeld M, Sievers HH. The influence of a nonlinear resistance element upon in vitro aortic pressure tracings and aortic valve motions. ASAIO J. 2004;50:498-502.

8. ISO 5840. Cardiovascular implants — cardiac valve prosthesis. 3rd ed. Genf, Switzerland: International Organization of Standardization; 1996.

9. Butterfield M, Fisher J, Lockie KJ, Davies GA, Watterson K. Frame mounted porcine valve bioprosthesis: preparation with aortic root dilatation. J Thorac Cardiovasc Surg. 1993;106:1181-8.

10. Robicsek F, Thubrikar MJ. Role of sinus wall compliance in aortic leaflet function. Am J Cardiol. 1999;84:944-6.

11. Sripathi VC, Kumar RK, Balakrishnan KR. Further insights into normal aortic valve function: role of a compliant aortic root on leaflet opening and valve orifice area. Ann Thorac Surg. 2004;77:844-51.

12. Furukawa K, Ohteki H, Cao Z-L, Narita Y, Okazaki Y, Ohtsubo S, et al. Evaluation of native valve-sparing aortic root reconstruction with direct imaging_reimplantation or remodeling? Ann Thorac Surg. 2004;77:1636-41.

13. Leyh RG, Schmidtke C, Sievers HH, Yacoub MH. Opening and closing characteristics of the aortic valve after different types of valve-preserving surgery. Circulation. 1999;100:2153-60. 